\title{
Bruxism in children: causes and solutions
}

\begin{abstract}
Why do children grind their teeth when they are asleep? Is bruxism an unconscious reaction to stress and anxiety or due to dental problems or even other diseases? What could be the causes of teeth grinding in children? Bruxism is a parafunctional involuntary activity that can have a variety reasons. It is a common phenomenon in childhood which is known as a type of sleep disorder. This disorder is seen in both primary and secondary types. In children, certain factors such as mouth breathing, enlargement of the tonsils, high filling restorations, the risk of some diseases, nutritional deficiencies, allergies, intestinal parasite, endocrine disorders, and psychological factors such as anxiety causes bruxism, are involved. The common symptoms of this problem can be listed as crown fractures, loosening of the teeth, tooth loss, tooth decay, oral and maxillofacial problems, headaches, TMJ pain and tooth sensitivities. About ways to eliminate teeth clenching, dentists have different views and in this respect, they still have not been able to find a suitable solution. But the use of a series of recommendations can be effective. Since child's bruxism is a natural reaction to grow, it cannot be prevented in the most cases. On the other hand, stress-induced bruxism is preventable.
\end{abstract}

Keywords: bruxism, parafunctional activity, sleep disorder, stress, anxiety, depression, tooth grinding
Volume 9 Issue 2 - 2018

\section{Karimi M}

Internal Auditor \& Documentations at Apple Dental Clinic, Iran

Correspondence: Karimi M, Internal Auditor \&

Documentations at Apple Dental Clinic, Iran,

Emaildrmokarimi@yahoo.com

Received: January 12, 2017 | Published: March 29, 2018

\section{Introduction}

Jaw clenching is very common in children up to 5 years at night. ${ }^{1,2}$ It is sometimes very weak and sometimes severs enough to wake up the parents and siblings. Most often, it causes distress and worries the parents. If the problem is severing, it may cause dental lesions and malocclusions in the future. Teeth grinding can also be caused due to local, systemic and psychological factors. This disorder is seen in both primary and secondary types. In children, usually has been observed in two periods of primary and permanent tooth eruption, and it often disappears in adolescence. To stop or treat bruxism, a series of specific recommendations is proposed that can be effective. At the Apple Dental Clinic, these are recommended to parents of children and adolescents. After a period of application of these tips, the results have been convincing.

\section{Types of bruxism}

Primary: In this type, there are no specific medical causes.

Secondary: When this parafunctional oral activity ${ }^{3}$ is associated with disorders such as psychiatric disorders (anxiety and depression), ${ }^{4-6}$ drugs or some diseases like Parkinson ${ }^{7}$ or respiratory diseases like apnea. ${ }^{5}$ The second type can usually be resolved by the treatment of the underlying disorder.

\section{Bruxism period}

Childhood jaw clenching is often treated on its own either by growth of the jaws or entering into adolescence. In most cases, when children lose their primary teeth, bruxism will be insignificant or will be completely faded. However, a small number of children continue grinding their teeth into adulthood. If the cause of is stress, this action will be continued as long as the problem is not resolved.

\section{Causes}

Pediatric Dentistry references books refer to these effective factors causing tooth clenching. These include the following:

A. Local factors: occlusal interferences, high filling restorations or poor restorations, irritating dental status

B. Systemic factors: parasitic and digestive diseases, malnutrition and nutritional deficiencies, allergies, endocrine disorders

C.Psychological factors: Personality disorders and increased stress

Irregular and crowded teeth and insufficient contact between the upper and lower teeth are the justified factors which explain the causes of bruxism.

Unconscious habits such as nail biting, chewing pencils and pen can be effective in jaw clenching. ${ }^{9}$ In some cases, having large tonsils that cause upper airway obstructions, have been considered as the involved factor. Bruxism risk factors are including factors such excessive anxiety, beverage containing caffeine (coffee, chocolate and cola), cigarettes, ${ }^{10}$ narcotics and psychotropic drugs. ${ }^{11}$ Some hyperactive children are also suffering from this disorder. Sometimes, children who have mental retardation, ${ }^{9}$ cerebral palsy, ${ }^{7,12}$ or using certain drugs, are at risk of bruxism. More surprisingly, given birth to the second child and unconscious and too much attention to that child is known as one of the causes of jaw clenching. Siblings or parents argument or fighting or problems in school and kindergarten are other factors which are considered.

\section{Signs and symptoms}

Bruxism usually has no specific symptoms but it may affect on the teeth surfaces area to that extent causes damaging the tooth enamel. The loss of tooth enamel may cause tooth sensitivities, cracked teeth and even tooth crown fractures. ${ }^{13}$ In the posterior teeth, attrition of the cusp is sometimes obvious. Of course, in some cases, other symptoms may occur such as headache, toothache, tooth mobility, gingival recession, atypical facial pain ${ }^{14}$ widening periodontal ligament, ${ }^{13}$ 
ulcers or pain in the cheeks, ${ }^{8,15}$ clicking sound upon opening and closing the mouth, TMJ pain ${ }^{13}$ sever tooth sensitivities ${ }^{13}$ and tongue's deformation. ${ }^{12}$ Inflammation and swelling can be seen on the side of the lower jaw due to teeth locking on each other. Jaw problems such as temporomandibular joint diseases are another sign that can be mentioned. It should be noted that most children who have this oral parafunctional activity, do not necessarily have any TMJ disorder unless they change into sever bruxers.

\section{Specific recommendations}

What should we do? Is there a cure for that? In order to prevent tooth clenching, one must first look for the problems that exist in the home and family and then find the solutions for them. The cause of teeth grinding at night or sleeping time have to be searched in child's discomfort and psychological pressure of those children who are unable to express their feelings; or have not responded to our surroundings. For example, in many cases it has been seen that child's mother has left her in sleep and has gone to work. When that child wakes up and her mother is not present at home, it is obvious that in such circumstances, that child would panic and would experience stress and fears. I refer here to numbers of important recommendations that I have been emphasizing to parents in our clinic for many years.

1. Reducing stress in children before bed time

2. Massage and stretching exercises for muscle relaxation of children

3. Reading the book before going to bed

4. Drinking plenty of water before sleeping

5. Listening to light and soul music

6. Put the warm towel on the cheeks of children

7. Preventing children from chewing pen or pencil

8. Prohibiting them from chewing gum during the day

9. Examination of teeth restorations of children by pediatric dentist

\section{Application of SS crown}

11. Making night guard under supervision of pediatric dentist if it is necessary

I recommend use of a technique which may not seem important. In Apple Dental Clinic, I suggested this method to the parents that have been effective in some way. Let children bite into cucumber, celery stalk, fresh carrots or an apple before sleep. This action causes fatigue in the jaws; hence, it prevents jaw muscle contraction. This treatment is useful especially for those kids who have nocturnal bruxism. Before sleeping, parents should embrace and cuddle their children and then ask them to talk about any fear, horror or resentment that bothering them. In my opinion, this definitely helps parents to keep bad memories away them. Going to the bedroom and putting them to sleep should be in peacefully and free from any discomfort. The correct daily behavior of parents, siblings, close relatives and also environmental factors affect the resolution of bruxism. If the problem is caused by jaw disorder, it can be remedied at an early age of orthodontic treatment. For this disorder in children, medication is usually not recommended. If there is a need to take drugs, it must be prescribed by a physician.

\section{Conclusion}

Although bruxism can be caused by stress or anxiety, but most often occurs during sleep because of crooked teeth, tooth loss and supernumerary teeth. The reason for the formation of this oral parafunctional activity is unknown but one may argue that crowded teeth, the arrangement of teeth out of the occlusion, inappropriate contact between the upper and the lower teeth, diseases such as malnutrition, nutritional deficiencies, allergies and endocrine disorders, and psychological factors like stress and anxiety can lead to bruxism in children. However, after tooth eruption, this habit is lost in most cases. If tooth clenching is diagnosed early, it can be treated by knowing the primary causes of this problem. But, if the treatment is late and bruxism is become a habit, then, it must be treated with the method of modification's habit.

\section{Acknowledgment}

None.

\section{Conflict of interest}

None.

\section{References}

1. Kuch EV, Till MJ, Messer LB. Bruxing and Non-bruxing children: A comparison of their personality traits. Pediatr Dent. 1979;1(3):182-7.

2. Sari S, Sonmez H. The relationship between occlusal factors and bruxism in permanent and mixed dentition in Turkish children. J Clin Pediatric Dent. 2001;25:191-194.

3. Wassell R, Naru A, Steele J, et al. Applied occlusion. London: Quintessence; 2008:26-30.

4. Lobbezoo F, Van Der Zaag J, Naeije M. Bruxism: its multiple causes and its effects on dental implants - an updated review. J Oral Rehabil. 2006;33(4):293-300.

5. Lobbezoo F, Van Der Zaag J, Naeije M. Bruxism: its multiple causes and its effects on dental implants - an updated review. J Oral Rehabil. 2006;33(4):293-300

6. Manfredini D, Lobbezoo F. Role of psychosocial factors in the etiology of bruxism. J Orofac Pain. 2009;23(2):153-66.

7. Bruxism/Teeth grinding. Mayo Foundation for Medical Education and Research. 2009.

8. Yldesley WR, Field A, Longman L. Tyldesley's Oral medicine. 5th ed. Oxford: Oxford University Press; 2003;195.

9. Cawson RA, Odell EW, Porter S. Cawsons essentials of oral pathology and oral medicine. 7th ed. Edinburgh: Churchill Livingstone. 2002; 6-366.

10. Ashroftt GW, Eccleston D, Waddell JL. Recognition of amphetamine addicts. Br Med J. 1965;1(5426):57.

11. Amir I, Hermesh H, Gavish A. Bruxism secondary to antipsychotic drug exposure: a positive response to propranolol. Clin Neuropharmacol. 1997;20(1):86-89.

12. Heasman P. Master Dentistry Vol I: Restorative dentistry, paediatric dentistry and orthodontics. 2nd ed. Edinburgh: Churchill Livingstone; 2008:177.

13. Macedo, Cristiane R, Machado MAC, et al. Pharmacotherapy for sleep bruxism". Cochrane Database of Systematic Reviews. John Wiley \& Sons, Ltd; 2009. 
14. International classification of sleep disorders, revised: Diagnostic and coding manual." (PDF). Chicago, Illinois: American Academy of Sleep Medicine; 2001.
15. Scully, Crispian. Oral and maxillofacial medicine: the basis of diagnosis and treatment. 2nd ed. Edinburgh: Churchill Livingstone; 2008;291-382. 